\title{
Assessment of Mass Comparator Sensitivity
}

\author{
Christian Müller-Schöll \\ Mettler-Toledo GmbH, Im Langacher 44, 8606 Greifensee, Switzerland
}

\section{ABSTRACT}

For the calibration of weight pieces, the evaluation of comparator sensitivity and its associated uncertainty component is essential. Yet, there is not much documented guidance on how to assess these values. This paper proposes a procedure for assessing, evaluating and optimizing mass comparator sensitivity.

\section{Section: RESEARCH PAPER}

Keywords: mass calibration; comparator sensitivity; uncertainty; mass comparator; OIML R111

Citation: Christian Müller-Schöll, Assessment of Mass Comparator Sensitivity, Acta IMEKO, vol. 6, no. 4, article 15, December 2017, identifier: IMEKO-ACTA06 (2017)-04-15

Editor: Paolo Carbone, University of Perugia, Italy

Received January 29, 2016; In final form November 18, 2017; Published December 2017

Copyright: (C) 2017 IMEKO. This is an open-access article distributed under the terms of the Creative Commons Attribution 3.0 License, which permits unrestricted use, distribution, and reproduction in any medium, provided the original author and source are credited

Funding: Mettler-Toledo GmbH, Greifensee, Switzerland

Corresponding author: Christian Müller-Schöll, e-mail: christian.mueller-schoell@mt.com

\section{INTRODUCTION}

When calibrating weight pieces, the difference between the unknown weight and the reference weight is calculated from the indications of the mass comparator display (or the data interface signal). This difference is then used to calculate the conventional mass of the unknown weight. For common industrial and laboratory applications, weight pieces are calibrated in conventional mass [1] and electronic balances and mass comparators also indicate in this conventional unit.

The term sensitivity is defined in [2] as "quotient of the change in an indication of a measuring system and the corresponding change in a value of a quantity being measured" $[2,4.12]$. According to this definition the general equation for sensitivity of a mass comparator is

$S=\frac{\Delta I}{\Delta m_{c}}$

with change in indication $\Delta I$ and conventional mass of a weight $\Delta m_{c}$ (and not the reciprocal value as is sometimes used, e.g. in [3] and [7]).

The general assumption is that for electronic balances and mass comparators, the difference $\Delta I$ of the indications is equal to the difference in conventional mass $\Delta m_{c}$. This corresponds to a sensitivity equal to 1 .

However, there are sources that suggest that this is not always the case. In earlier days, it was apparently usual that comparators with an optical scale did not necessarily indicate correct mass differences. Therefore, [3, SOP4] and [1, C.4.1.2], for example, describe weighing cycles for mass calibrations including a sensitivity weight $(\mathrm{S})$, e.g. of the form " $\mathrm{A}-\mathrm{B}$ $\mathrm{B}+(\mathrm{S})-\mathrm{A}+(\mathrm{S})$ ". With these cycles, the sensitivity is determined in every single weighing cycle to convert the scale divisions into a mass difference.

In Section 2 we will review existing literature on this subject. Sections 3 and 4 deal with uncertainty questions. In Sections 5 through 7 we derive a procedure for assessing comparator sensitivity based on optimized uncertainty. Section 8 presents a verification through experimental data. The conclusions finally summarize the findings.

\section{REVIEW OF THE LITERATURE}

\subsection{Testing Procedures for Sensitivity}

According to some sources in the literature, it is not necessary to determine sensitivity in every weighing cycle for modern electronic comparators:

- "A sensitivity weight is not required if the electronic mass comparator that is used has been tested (with supporting data available) to determine that the balance has sufficient accuracy..." [3, GMP 14]. The uncertainty of this assumption shall be included as an uncorrected systematic error in the uncertainty budget and acceptable limits are "2\%" [3, SOP8]. 
- Kochsiek et al. mention in the German edition of their book "Massebestimmung" [4] (translated to English: "mass determination") that for a "frequently adjusted electromagnetically compensated balance" sensitivity $S$ shall be assumed to be 1 and an uncertainty in $S$ shall be assumed to be less than $5 E-04$ or may even be neglected, especially when using auxiliary weights ...”.[4]. However, it remains unclear how to adjust the comparator so that it fulfils the condition of being "adjusted", nor are there any suggestions as to the accuracy and thus uncertainty of the adjustment procedures, nor as to how often an adjustment must take place to fulfil the requirement of being "frequently adjusted". It is interesting to note that the cited numerical value $(5 E-04)$ is omitted in the (later) English edition of the same book [5].

- Chapter C.6.4.2 of [1] requires that an uncertainty component for sensitivity be included in the budget when calibrating weights, but gives no specific guidance on how to assess the value of this component and especially on how to select a proper sensitivity test weight regarding its size and its calibration quality.

In general, there are two ways to treat sensitivity: One is to assume ideal sensitivity and set a limit for the deviation of the sensitivity from "1" and test and verify the sensitivity against that limit. This limit value is considered in the uncertainty budget as an uncorrected error. The other one is to evaluate a value for sensitivity, correct all balance readings with this value, calculate the uncertainty of this correction and have this contribute to the uncertainty budget (which results in a smaller uncertainty but requires a higher calculation effort).

Since mass calibrations involve a significant amount of calculations, it is common practice today to use at least software spreadsheets or even dedicated software for the calculations. If this is the case, the sensitivity value can easily be incorporated as a correction in the calculation of the mass differences and its uncertainty will then contribute to the uncertainty calculation.

In some commercially available calibration software (e.g. Scalesnet, McLink), a numerical value of sensitivity is determined in a separate test for each comparator. But for its uncertainty, the uncertainty value given in [4] is frequently used without alteration. Where the mass difference between the calibrated weight and the reference weight becomes large, this might have significant influence on the final combined calibration uncertainty of the weight piece under test. One result of this paper is to answer the question whether this worst case estimation of a sensitivity uncertainty of $5 E-04$ is justified.

\subsection{Test Weights for Sensitivity Testing}

There seems to be common understanding that sensitivity of mass comparators is tested with a "small" weight: Reference [3, SOP 2] mentions a "small weight", [3, SOP 34] mentions a maximum of $0.5 \%$ of balance capacity, while [3, GMP 10] mentions a maximum of $1 \%$ of balance capacity. It remains unclear if "balance capacity" means the full load value or the electrical weighing range in this context. No standard method for the selection of the sensitivity test weight appears to be available.

\section{ASSESSMENT OF SENSITIVITY}

The VIM [2] defines adjustment of a measuring system as "set of operations carried out on a measuring system so that it provides prescribed indications corresponding to given values of a quantity to be measured".

Today's mass comparators with electromagnetic force compensation are equipped with provisions for self-adjustment. These consist of one (or more) internal weight piece(s) and an algorithm which can be time and/or temperature controlled or manually triggered. Furthermore, external mass calibration software allows for an additional "adjustment" of the reading by applying a sensitivity factor in the processing of the value that was read from the comparator output. In this light, we consider the calibration software a part of the "measuring system" together with the comparator (see Figure 1), and the application of a sensitivity value is considered an adjustment of this system.

The sensitivity factor applied by the software is gained from the following test procedure: The comparator is loaded with a pre-load (between zero and nominal load) which brings the comparator into a typical working range and working state. Then a test is carried out using an "ABBA" cycle which starts with the mentioned pre-load ("A"), then a calibrated sensitivity test weight with conventional mass $\Delta m_{c, S}$ is added (" $\mathrm{B}$ "), this step is repeated (" $\mathrm{B}$ ") and finally the test weight is removed ("A"). From the calibration value of the test weight and the calculated, buoyancy-corrected difference of comparator indication during the test $\Delta I_{S}$, the sensitivity value $S$ is calculated according to (1). (Subscripts upper case " $S$ " are used in this paper to indicate the context of a sensitivity test).

Once this sensitivity value is determined, it must be used for any further processing of a reading of that comparator. We assume here that the self-adjustment procedure of the comparator is run about once a day, so that any climate-induced changes (air density, temperature) and their effect on the comparator sensitivity are negligible, this means that the sensitivity of the comparator stays "the same" over time (but will not necessarily be 1 exactly).

\section{UNCERTAINTY OF SENSITIVITY}

\subsection{General Considerations}

Given the procedure above and (1) for the calculation of sensitivity, we find the following sources of uncertainty when determining $\mathrm{S}$ from a sensitivity test:

- Readability of the comparator $u_{\text {read }}$

- Repeatability of the comparator $u_{\text {repeat }}$

- Calibration uncertainty of the sensitivity test weight $u_{\text {weight }}$

\section{Measuring System: delivers processed value}

Software (external of comparator): processes indication value with a factor

Comparator:

delivers indication value

Figure 1. The measuring system. 
The uncertainty in $S$ can thus easily be derived as:

$u_{S}^{2}=\left[\frac{1}{\Delta m_{c, S}} \times u_{\text {read }}\right]^{2}+\left[\frac{1}{\Delta m_{c, S}} \times u_{\text {repeat }}\right]^{2}+$

$+\left[\frac{\Delta I_{S}}{\Delta m_{c, S}{ }^{2}} \times u_{\text {weight }}\right]^{2}$

with the components

$u_{\text {read }}=\frac{d}{2} \times \frac{1}{\sqrt{3}}$

for an ABBA cycle (derived from the calculation of an ABBA difference) and with comparator indication readability $d$, $u_{\text {repeat }}=\frac{s}{\sqrt{n}}$

with the repeatability standard deviation $s$ of the comparator and the number of cycles of the sensitivity test $n$ and

$u_{\text {weight }}=\frac{U_{\text {cal }}}{k}$

with coverage factor $k$, taken from the calibration certificate of the sensitivity test weight.

For simplification of the uncertainty calculation (only) we set $\Delta I_{S}=\Delta m_{c}$ so that (2) becomes for a sensitivity test:

$u_{S}^{2}=\left[\frac{1}{\Delta m_{c, S}} \times \frac{d}{2 \times \sqrt{3}}\right]^{2}+\left[\frac{1}{\Delta m_{c, S}} \times \frac{s}{\sqrt{n_{S}}}\right]^{2}+\left[\frac{1}{\Delta m_{c, S}} \times \frac{U_{c a l}}{2}\right]^{2}$

Please note that since $S$ is a relative number, its uncertainty $u_{S}^{2}$ is also a relative number while its uncertainty contributors are in mass units.

\subsection{Uncertainty of Conventional Mass - Connection to OIML R111}

OIML R111 [1] requires an uncertainty contribution $u_{s}$ (note lower case "s" in the subscript) to be estimated that, as a component of the balance uncertainty $u_{b a}$, accounts for the uncertainty of the sensitivity in the calculation of conventional mass. In our case, where a factor $S$ is used to calculate the conventional mass difference according to (1), this uncertainty component of conventional mass is (with sensitivities $S$ close to 1):

$u_{S}^{2}=\left[\frac{\Delta I}{S^{2}} \times \mathrm{u}_{S}\right]^{2} \approx\left[\Delta I \times \mathrm{u}_{S}\right]^{2}$

Note that $u_{s}^{2}$ (with lower case subscript $s$ ) is taken from [1] while the last term (with upper case $S$ ) is our concept.

\section{INITIAL CHOICE OF A SENSITIVITY TEST WEIGHT}

As has been mentioned above, there is only little literature available on mass comparator sensitivity. The publication of R. Davis [6] appears to have been written in the light of comparators with optical scales and a sensitivity assessment in every mass calibration cycle and therefore provides only a rough direction for today's questions. Lee Shih Mean's paper [7] focuses on a very special problem (the calibration of stainless steel against Pt-Ir standards and evaluating true mass) and thus has its own reasons for the choice of the weight size. All sources, however, agree in the general idea that the weight should be "small" and in the magnitudes of the weighing differences that will be obtained. The reason is probably, that this procedure tries to approximate an ideal differential sensitivity $\frac{\partial I}{\partial m_{c}}$ from the test with finite values $\frac{\Delta I_{S}}{\Delta m_{c, S}}$ thus avoiding any influence of non-linearities in the characteristic curve of the comparator.
As a first practical assumption, we chose calibrated test weights with a nominal value of about 100 times the readability $d$ of the comparator, but not smaller than the smallest OIMLweight which is $1 \mathrm{mg}$. The weights are made of stainless steel to avoid any complications arising from buoyancy effects. As test objects we chose the manual comparators in the mass calibration laboratory of METTLER TOLEDO, accredited as SCS 0032. (Table 1).

We further assume for simplicity reasons that the sensitivity test weights were calibrated with an uncertainty $(k=2)$ of one third of the MPE of class $E_{1}$ according to [1].

Thirdly, we used "datasheet repeatablities" for our calculations and $n_{S}=1$ repetitions for the sensitivity test. For each mass comparator used in our laboratory, we calculated the uncertainty of the sensitivity as given in (3).

This revealed some unexpected results. Figure 2 shows the sensitivity uncertainties $(k=1)$ for each comparator type listed in Table 1. We note the following important findings:

- Although we applied the same basic idea for the choice of the sensitivity test weight, the differences of the sensitivity uncertainties between the comparators were of about 3 magnitudes, ranging from 6 E-04 to 2 E-02.

- The maximum uncertainty value observed (2 E-02) was significantly higher than the simplified value of 5 E-04 from the literature although our procedure uses a correction.

We conclude that a general assumption of an uncertainty of comparator sensitivity of $5 \mathrm{E}-04$ (as can be found in the literature) is not justified.

\section{VARIATION AND OPTIMIZATION OF PARAMETERS}

\subsection{Optimization to reach an Uncertainty of $5 \mathrm{E}-04$}

Further investigation of the uncertainty budget of the sensitivity revealed that in most cases (and especially in the case

Table 1. Mass comparators, datasheet repeatabilities and readabilities and test weights nominal values of "initial choice".

\begin{tabular}{|c|c|c|c|c|c|c|c|}
\hline 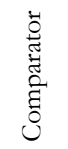 & 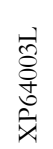 & 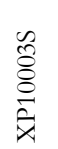 & 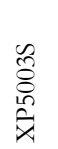 & 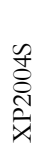 & $\begin{array}{l}\stackrel{n}{8} \\
\frac{\pi}{y} \\
z\end{array}$ & $\begin{array}{l}\stackrel{8}{\vec{x}} \\
\frac{\pi}{4}\end{array}$ & $\begin{array}{l}D \\
\not ்\end{array}$ \\
\hline $\begin{array}{l}d \\
(\mathrm{mg})\end{array}$ & 5 & 1 & 1 & 0.1 & 0.01 & 0.001 & 0.0001 \\
\hline $\begin{array}{l}S \\
(\mathrm{mg})\end{array}$ & 8 & 1 & 0.8 & 0.1 & 0.02 & 0.003 & $\begin{array}{c}0.0003 \\
5 \\
\end{array}$ \\
\hline $\begin{array}{l}m_{c, S} \\
(\mathrm{mg})\end{array}$ & 500 & 100 & 100 & 10 & 1 & 1 & 1 \\
\hline
\end{tabular}

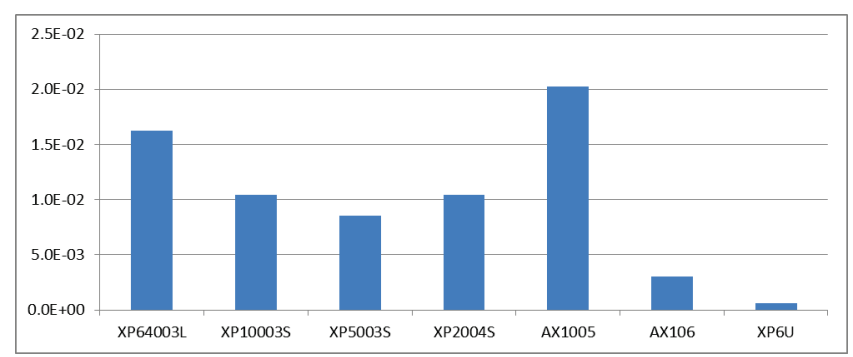

Figure 2. Sensitivity uncertainties based on initial choice of the sensitivity test weight $(k=1)$. 
of the high values identified above), the dominant contributor to the uncertainty budget was the influence of repeatability (which is the second component in (3)). The equation suggests that this contributor could be reduced by an increase in the number of weighing cycles used in the adjustment. However, this has little effect since it is not practical to use more than about 5 ABBA cycles. (For this publication, we will continue to use datasheet repeatabilities. However, it is obvious that using individually determined repeatabilities (which are usually smaller) will have a significant, improving impact on the uncertainty.)

Re-visiting equation (3), we find that the nominal value of the chosen sensitivity test weight influences all three uncertainty contributors. This opens the door to optimizing the sensitivity uncertainty by adjusting number of cycles and nominal values of the test weights. Increasing the nominal weight value will lead to smaller sensitivity uncertainties. A massive increase would, however, violate the principle of "small" sensitivity test weights (as explained above in Section 2.2), so we prefer to keep nominal values small. Additionally, we will only use nominal values that are specified in [1].

With these restrictions, we iteratively increased the nominal values in the sensitivity test weight with the aim of reducing uncertainties of all sensitivities to approximately $5 \mathrm{E}-04$ or less (which is the literature value). In order to keep the procedures easy to understand for all laboratory personnel, we fixed the number of weighing cycles for the sensitivity test to $n_{S}=3$ ABBA cycles for all types of comparators. The new values are shown in Figure 3. Please note the difference in y-axis scale compared to Figure 2.

These results were achieved using the sensitivity test weights for the comparator models as shown in Table 2. The nominal values of these weights are below the maximum value of $0.5 \%$ of balance capacity as stipulated in [3], so this choice does not violate the concept of a "small" weight.

\subsection{Further Optimization to reach Smaller Uncertainties}

By increasing the number of cycles and by increasing the nominal values of the weight pieces, it is possible to reach

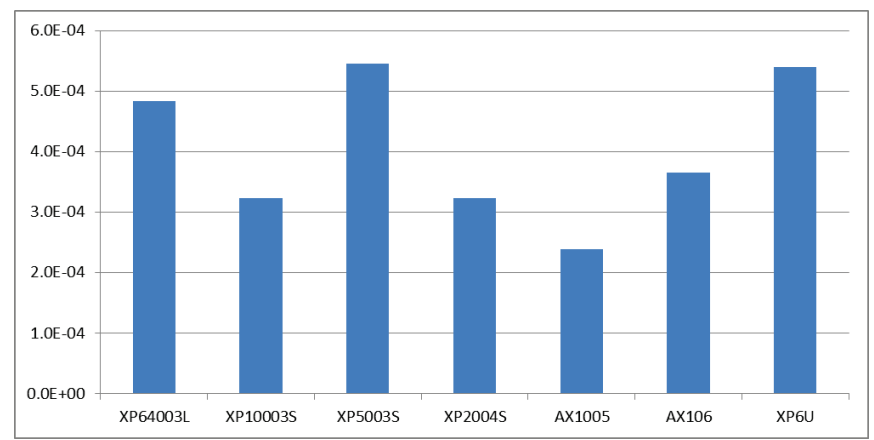

Figure 3. Sensitivity uncertainties with optimized procedure $(k=1)$.

Table 2. Mass comparators and test weight nominal values for optimized sensitivity.

\begin{tabular}{|c|c|c|c|c|c|c|c|}
\hline 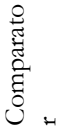 & 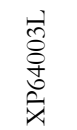 & $\begin{array}{l}\frac{n}{\tilde{\delta}} \\
\delta \\
\frac{\delta}{x}\end{array}$ & 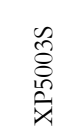 & 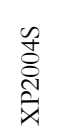 & $\begin{array}{l}\stackrel{0}{8} \\
\frac{1}{4} \\
\frac{1}{4}\end{array}$ & $\begin{array}{l}\stackrel{\circ}{\not} \\
\stackrel{x}{\&}\end{array}$ & $\begin{array}{l}\text { Ð } \\
\text { ڤ્ર }\end{array}$ \\
\hline $\begin{array}{l}m_{c} \\
(\mathrm{mg})\end{array}$ & 10000 & 2000 & 1000 & 200 & 50 & 5 & 1 \\
\hline
\end{tabular}

values for $u_{s}$ of e.g 1 E-04.

However, with an attempt to reach 1 E-05 (still using datasheet repeatabilities), the necessary test weights approach the " $0.5 \%$ of capacity limit" (see above) and thus are no longer considered "small".

\subsection{Variation of Sensitivity Test Weight Accuracy}

Except for microbalance comparators, the calibration uncertainty of the sensitivity test weight $U_{c a l}$ has little influence on the uncertainty in $S$. So using weights calibrated in $\mathrm{E}_{2}$ quality instead of $\mathrm{E}_{1}$ weights is possible without major disadvantage.

\section{GENERAL COOKBOOK PROCEDURE FOR ASSESSING OPTIMIZED COMPARATOR SENSITIVITY AND ITS UNCERTAINTY}

The following procedure for assessing sensitivity uncertainty can be derived from the considerations above:

1. Set a maximum acceptable value for sensitivity uncertainty (e.g. $5 \mathrm{E}-04$ or $1 \mathrm{E}-05)$.

2. Set a number of ABBA cycles for the sensitivity test, use (3) and iteratively increase the nominal weight value until the above condition is fulfilled for the comparator concerned.

3. Execute sensitivity test and apply the value found for $S$ to all future readings.

4. Use the value of $u_{s}$ for the uncertainty estimation of mass calibrations according to [1].

\section{EXPERIMENTAL VERIFICATION}

In order to verify the theoretical ideas outlined above, we have done several tests. One series of tests was done on an XP2004S comparator balance. We have tested different weight sizes and numbers of test cycles. The results are found in Figure 4: the diagram shows 3 series of sensitivity values and their calculated uncertainties. The first series was done with a $100 \mathrm{mg}$ sensitivity test weight and one cycle, while the second series was done with a $500 \mathrm{mg}$ weight and three cycles. The third series was done with a $1 \mathrm{~g}$ test weight and three cycles. The picture confirms the theoretical considerations:

- As could be expected, the variability of the values gained with higher uncertainty is bigger. The larger the test weights and the number of cycles, the smaller the variability.

- Most data points are consistent to each other: An En-Test shows that most data points are consistent with every other one, especially the points gained with more than one cycle (i.e. $n_{S}>1$ ) show excellent agreement with each other.

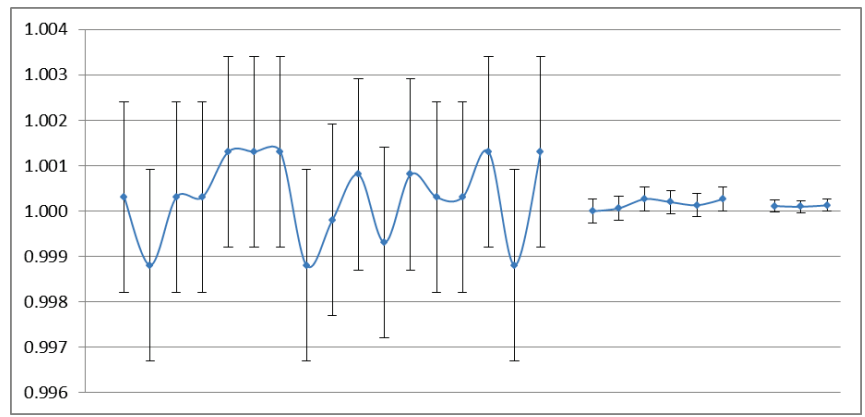

Figure 4. Three series of sensitivity test points together with calculated uncertainties $(k=2)$. Nominal values and number of cycles for each series: $100 \mathrm{mg}(\mathrm{n}=1), 500 \mathrm{mg}(\mathrm{n}=3), 1 \mathrm{~g}(\mathrm{n}=3)$. 


\section{CONCLUSIONS}

The sensitivity of mass comparators and its associated uncertainty are both values with important significance in the field of weight piece calibration. The literature does not provide much guidance neither on procedures to be used for assessing sensitivity and its uncertainty nor on the selection of suitable weights for sensitivity testing. We have presented a procedure for assessing and the mathematics for evaluating sensitivity and its associated uncertainty. By means of iterative application, a weight for a sensitivity test can be selected so that relative sensitivity uncertainties $u_{s}$ of e.g. 1 E-04 are achieved with the prerequisite that a correction for sensitivity is applied.

\section{REFERENCES}

[1] OIML: "OIML R111-1:2004: Weights of classes $E_{1}, E_{2}, F_{1}, F_{2}$, $\mathrm{M}_{1}, \mathrm{M}_{1-2}, \mathrm{M}_{2}, \mathrm{M}_{2-3}$ and $\mathrm{M}_{3}$ - Part 1: Metrological and technical requirements": www.oiml.org.
[2] JCGM 200: 2012: "International vocabulary of metrology - Basic and general concepts and associated terms (VIM) 3rd edition": www.bipm.org.

[3] NIST: IR 6969: www.nist.gov.

[4] Kochsiek M, Glaeser M, Massebestimmung, VCH, Weinheim, 1997

[5] Kochsiek M, Glaeser M (eds.). Comprehensive Mass Metrology, Wiley-VCH, Berlin, 2000.

[6] R. Davis: "Note on the Choice of a Sensitivity Weight in Precision Weighing", Journal of Research of the National Bureau of Standards, Volume 92, Number 3, pp 239-242, May-June 1987.

[7] S. M. Lee, R. Davis, L. K. Lim: "Calibration of a $1 \mathrm{~kg}$ Stainless Steel Standard with respect to a $1 \mathrm{~kg}$ Pt-Ir Prototype: A Survey of Corrections and Their Uncertainties", Asia-Pacific Symposium on Mass, Force and Torque (APMF 2007), Oct 24-25 2007.

Certain commercial equipment, instruments, or materials are identified in this paper in order to adequately describe the experimental procedure. Such identification does not imply recommendation or endorsement by the author, nor does it imply that the materials or equipment identified are the only or best available for the purpose. 